\title{
Adaptação transcultural da versão em português do Brasil do questionário Quality of Life Headache-Youth (QLH-Y)
}

\author{
Cross-cultural adaptation of the Brazilian Portuguese version of Quality of \\ Life Headache-Youth (QLH-Y) Questionnaire
}

Gabriela Almeida da Silva', Dayzene da Silva Freitas' ${ }^{7}$ Clarice Nicéas Barreto da Costa', Sheva Castro Dantas de Sousa', Marcelo Moraes Valença², Daniella Araújo de Oliveira',2

'Departamentos de Fisioterapia e ${ }^{2}$ Neuropsiquiatria, Universidade Federal de Pernambuco (UFPE), Recife, PE, Brasil

Silva GA, Freitas DS, Costa CN, Sousa SC, Valença MM, Oliveira DA. Adaptação transcultural da versão em português do Brasil do questionário Quality of Life Headache-Youth (QLH-Y).

Headache Medicine. 2013;4(2):45-53

\section{RESUMO}

Objetivos: Traduzir para a língua portuguesa, adaptar transculturalmente e validar o Quality of Life HeadacheYouth (QLH-Y), avaliando sua confiabilidade por meio da consistência interna dos seus itens, em uma amostra de adolescentes de ambos os gêneros, estudantes de escolas públicas na cidade de Recife. Método: $\bigcirc$ instrumento original foi traduzido do inglês para o português, gerando uma síntese consensual. A versão em português do instrumento foi retrotraduzida, e então desenvolvida uma versão pré-final para um teste de campo. Após a adequação do instrumento para o português, foi realizado um estudo piloto com 100 estudantes. Resultados: Os resultados encontrados na consistência interna da versão brasileira foram muito satisfatórios, com o valor de alfa de Cronbach de 0,906 (superior a 0,7 ) indicando um grau elevado de consistência interna. Conclusão: $A$ versão em português do QLH-Y apresenta uma consistência interna excelente e uma boa compreensão dos seus itens pela adaptação transcultural na cidade do Recife.

Palavras-chave: Adolescentes; Cefaleia; Transtornos de enxaqueca; Qualidade de vida

\section{ABSTRACT}

Objectives: To translate into Portuguese, culturally adapt and validate the Quality of Life Headache - Youth (QLH-Y), assessing its reliability through internal consistency of its items in a sample of adolescents of both sexes, attending public schools in the city of Recife. Methods: The original instrument was translated from English into Portuguese, generating a synthetic consensus. The Portuguese version of the instrument was back translated, and then developed a pre-final version for a field test. After the suitability of the instrument into Portuguese was conducted a pilot study with 100 students. Results: The findings of the internal consistency of the Brazilian version were very good, with the alpha value of 0.906 (greater than 0.7 ) indicating a degree high internal consistency. Conclusion: The Portuguese version of QLH $Y$ has an excellent internal consistency and a good understanding of its items for cross-cultural adaptation in the city of Recife.

Keywords: Adolescents; Headache; Migraine disorders; Quality of life 


\section{INTRODUÇÃO}

A cefaleia é uma das queixas somáticas mais frequentes relatadas entre crianças e adolescentes. (1) Pode causar um grande impacto na vida diária das crianças e dos pais, como nas atividades sociais, atividades com a família e absenteísmo escolar. ${ }^{(2)}$

A qualidade de vida é uma medida importante da efetividade do tratamento da cefaleia, que reflete o impacto da doença na avaliação subjetiva do paciente, no bem-estar emocional e funcionamento físico. ${ }^{(3-5)}$ Crianças com cefaleia apresentam menor qualidade de vida do que crianças saudáveis. Comparando com crianças que sofrem doenças crônicas (como reumatismo ou câncer), os valores de qualidade de vida foram semeIhantes no que diz respeito à deficiência escolar e funcionamento emocional. ${ }^{(6)}$

A prevalência da cefaleia tende a aumentar com o avançar da idade. (7) Um estudo realizado em São José do Rio Preto - SP(8) avaliou a prevalência e a frequência de cefaleia em escolares brasileiros. $\bigcirc$ resultado mostrou que $91,8 \%$ dos alunos tiveram cefaleia no último ano. Destes, $53,7 \%$ relataram que a dor acontecia frequentemente. Em relação ao gênero, $36,6 \%$ das meninas e $28,2 \%$ dos meninos queixavam-se de cefaleia pelo menos uma vez por semana. No caso da cefaleia diária, o número de meninas chega a quase o dobro dos meninos. Uma das causas prováveis dessa desigualdade entre os sexos em determinada faixa etária são as alterações hormonais. ${ }^{(9)}$ Em outro estudo realizado com adolescentes brasileiros, a frequência de cefaleia do tipo tensional foi maior no sexo feminino, enquanto que a migrânea ocorreu com mesma frequência entre homens e mulheres. ${ }^{(10)}$

Um estudo com crianças, realizado na Suécia, fez uma comparação entre dor nas costas e dor de cabeça, (11) observou-se que a dor de cabeça atrapalha mais a escola do que as dores lombares no que se refere às habilidades de concentração, ao uso de analgésicos ou ao absenteísmo escolar. ${ }^{(12)}$ Além disso, recentes pesquisas sugerem que crianças com dor de cabeça apresentam um risco maior de problemas psicológicos, incluindo sintomas de ansiedade e depressão. ${ }^{(13,14)}$ Crianças com dor de cabeça mostram menor participação social e mais queixas somáticas. Elas são menos felizes na escola e são mais ansiosas do que uma criança sem dor. ${ }^{(15)}$

Apesar de elevada, a cefaleia é frequentemente subestimada e subdiagnosticada na infância e adolescência, ${ }^{(10,16)}$ causando impacto na qualidade de vida.
Portanto, é necessária a elaboração de um instrumento validado e adaptado transculturalmente para a língua portuguesa, específico para adolescentes com cefaleia. A escolha de um instrumento que avalie a qualidade de vida possibilita a realização de comparações interculturais de intervenções e de diferentes estados de saúde com benefícios relativos de tempo e custo.

Na literatura existem alguns questionários específicos para avaliar a qualidade de vida em crianças e adolescentes com migrânea, dentre eles: o Migraine Specific Questionnaire (MSQ), o Migraine Specific Quality of Life Measure (MSQOL) e o Quality of Life Headache-Youth Questionnaire (QLH-Y). O QLH-Y é um instrumento desenvolvido para o uso em adolescentes com cefaleia ou migrânea ${ }^{(17)}$, o qual avalia a qualidade de vida em seis domínios: funcionamento psicológico, status funcional, funcionamento físico, funcionamento social, satisfação com a vida em geral e satisfação com a saúde. Os limites cronológicos da adolescência são definidos pela Organização Mundial da Saúde (OMS) entre 10 e 19 anos. ${ }^{(18)}$

Assim, este estudo tem como proposta traduzir para a língua portuguesa, adaptar e validar o Quality of Life Headache-Youth Questionnaire, avaliando a consistência interna de seus itens pela análise do alfa de Cronbach em uma amostra de adolescentes de ambos os gêneros, estudantes de escolas públicas na cidade de Recife.

\section{MÉTODOS}

Trata-se de um estudo metodológico, onde foi solicitada a autorização dos autores para a realização da validação e adaptação transcultural do Quality of Life Headache-Youth Questionnaire para a região da cidade do Recife-PE. Foram incluídos 130 adolescentes entre 10 a 19 anos de idade ( $14 \pm 2$ anos), de ambos os sexos, cursando do $6^{\circ}$ ano do ensino fundamental ao $3^{\circ}$ ano do ensino médio, matriculados em cinco escolas públicas estaduais de Recife, nos meses de janeiro a junho de 2012, conforme descrito na Figura 1. O início da coleta de dados do presente estudo se deu após a aprovação do Comitê de Ética em Pesquisa com seres humanos, com número CAAE 02043612.6.0000.5208. Os responsáveis pelos adolescentes assinaram o Termo de Consentimento Livre e Esclarecido. Foram excluídos do estudo alunos com histórico de doenças neuropsicológicas ou incapacidade cognitiva.

O instrumento utilizado foi o Quality of Life HeadacheYouth Questionnaire, um questionário que possui 71 itens, sendo 69 questões do tipo Likert e duas escalas visuais 
analógicas de satisfação com a vida em geral e com a saúde. Do item 1 ao item 55, existem quatro possíveis respostas: "Raramente ou nunca", "Às vezes", "Muitas Vezes" ou "Várias Vezes ou sempre". Do item 56 ao item 69, existem quatro possíveis respostas: "De jeito nenhum", "Pouco", "Mais ou menos" ou "Muito". As respostas são todas em relação à semana anterior. ${ }^{(17)}$

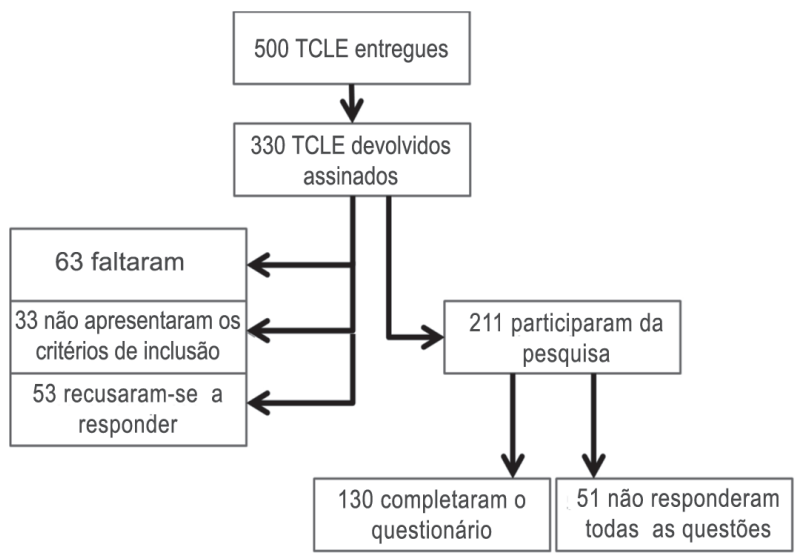

Figura 1 - Fluxograma de seleção da amostra, Recife, Pernambuco, 2012

processo de tradução e adaptação cultural foi realizado segundo a proposta de Beaton et al.,(19) através de várias etapas metodológicas: tradução, síntese, retrotradução, revisão por um comitê de especialistas, préteste e a verificação das propriedades psicométricas (Figura 2).

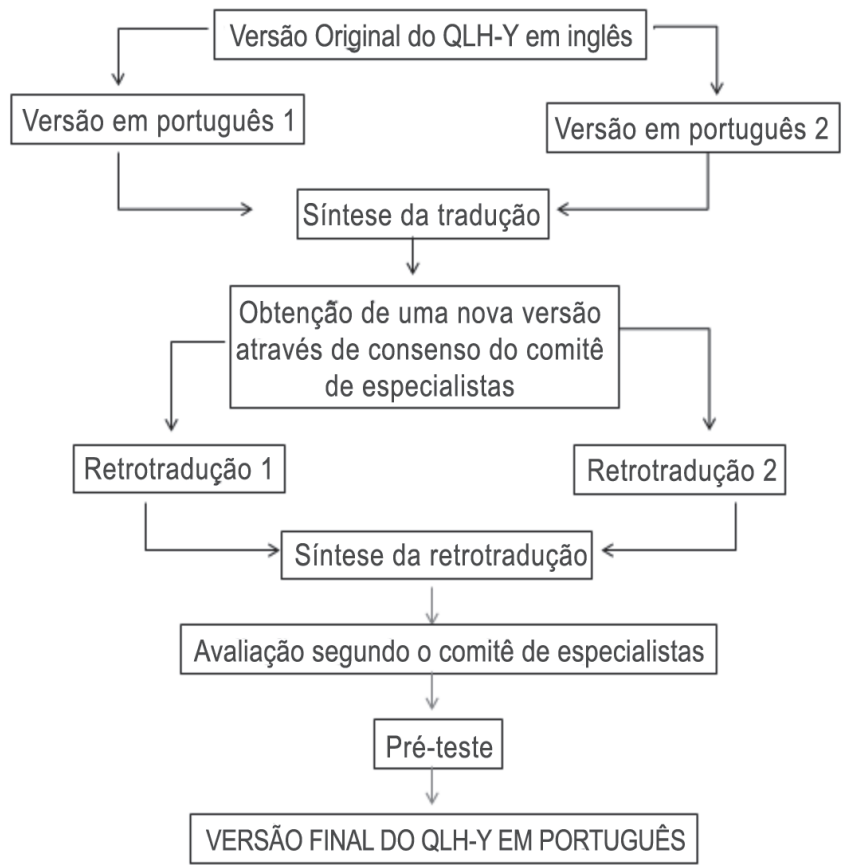

Figura 2 - Etapas do processo de tradução e adaptação transcultural de acordo com os critérios estabelecidos por Beaton et al., 2000. (20)
Na primeira etapa, o instrumento original foi traduzido do inglês para o português do Brasil por dois profissionais independentes bilíngues, ambos orientados sobre a população alvo em que o instrumento foi utilizado (adolescentes da rede pública de ensino).

Na segunda etapa, as versões foram comparadas entre os tradutores para identificar discrepâncias, gerando uma síntese consensual.

$\mathrm{Na}$ terceira etapa, a versão em português do instrumento foi traduzida para o inglês por outros dois tradutores independentes. A retrotradução assegura que a versão reflete o mesmo conteúdo da versão original. Os dois tradutores não estavam cientes nem informados dos conceitos explorados e possuíam o inglês como língua materna.

Na quarta etapa, foi realizada uma revisão por um comitê especialista, essencial para alcançar a equivalência semântica transcultural, consolidar todas as versões dos questionários e desenvolver uma versão pré-final para o teste de campo.

Na quinta etapa, a versão pré-final foi aplicada para a avaliação da compreensão e verificação da aceitabilidade do instrumento e para a realização de correções necessárias. $\bigcirc$ pré-teste foi realizado em uma amostra de 30 adolescentes, com idade 10 a 19 anos.

A análise semântica tem como objetivo verificar se todos os itens são compreensíveis para todos os membros da população à qual o instrumento se destina. Esses adolescentes comentaram as questões da versão de consenso, apontando dificuldades e sugerindo termos de mais fácil compreensão. A partir dessas sugestões, foi elaborada a versão final em português do Quality of Life Headache-Youth Questionnaire.

Após a adequação do instrumento ao idioma português do Brasil, foi realizado um estudo piloto com 100 estudantes, 51 do sexo feminino, estudantes de escolas públicas estaduais da região metropolitana do Recife, com idade entre 10 e 19 anos (14 14 ).

Para que a adaptação transcultural seja alcançada, é também necessário um estudo de equivalência de mensuração, com avaliação da medida de confiabilidade através do alfa de Cronbach para cada domínio e cada subescala. A consistência interna é determinada com uma única aplicação do instrumento, verificando-se a homogeneidade dos itens de modo que meçam as mesmas dimensões. ${ }^{(21)}$

Convém ressaltar que a interpretação do alfa de Cronbach relaciona-se a interpretações dadas por estimativas de confiabilidade baseadas no método "Split-half", 
já que representa a média de todos os coeficientes "Splithalf" para um dado instrumento.

Em geral, escalas com alfa de Cronbach maior que 0,70 são evitadas, pois indicam pouca confiabilidade. No entanto é válido lembrar que o valor de alfa de Cronbach tende a aumentar conforme o número de questões do instrumento.

O programa utilizado para a digitação dos dados e obtenção dos cálculos estatísticos foi o SPSS (Statistical Package for the Social Sciences) na versão 20.

\section{RESULTADOS}

No pré-teste foram avaliados 30 adolescentes (17 meninas) com idade entre 12 e 16 anos ( $12 \pm 2$ anos). No estudo piloto foram avaliados 100 adolescentes (51 meninas), com idade entre 12 e 18 anos (14 42 anos).

Durante o processo de tradução e adaptação do QLH-Y, alguns termos foram alterados com o objetivo de manter o mesmo significado da versão original. Tais ajustes foram descritos na Tabela 1. Os demais itens do Questionário não apresentaram necessidade de alterações nesta etapa do processo.

Tabela 1. Demonstrativo dos ajustes realizados na tradução do QLH-Y

\begin{tabular}{|c|c|c|c|}
\hline $\begin{array}{c}\mathrm{N}^{0} \mathrm{da} \\
\text { questão }\end{array}$ & $\begin{array}{l}\text { Versão } \\
\text { original }\end{array}$ & $\begin{array}{l}\text { Síntese da } \\
\text { tradução }\end{array}$ & $\begin{array}{l}\text { Versão } \\
\text { final }\end{array}$ \\
\hline 9 & Worthless & Sem valor & Insignificante \\
\hline 18 & Lively & Vigoroso & Ativo \\
\hline 21 & $\begin{array}{c}\text { If there were } \\
\text { activities at home } \\
\text { that we could all } \\
\text { do together, I also } \\
\text { took part }\end{array}$ & $\begin{array}{l}\text { Se houve } \\
\text { atividades em } \\
\text { casa, em que todos } \\
\text { nós pudéssemos } \\
\text { fazê-las juntas, eu } \\
\text { também participava }\end{array}$ & $\begin{array}{l}\text { Participei de } \\
\text { atividades na } \\
\text { minha casa em } \\
\text { que todos } \\
\text { puderam } \\
\text { participar }\end{array}$ \\
\hline 35 & I felt gloomy & $\begin{array}{l}\text { Eu me senti } \\
\text { depressivo }\end{array}$ & Eu me senti triste \\
\hline 50 & Muscle stiffness & rigidez muscular & tensão muscular \\
\hline
\end{tabular}

O valor do alfa de Cronbach para todo o questionário (69 questões) foi de 0,906. Os valores do alfa de Cronbach por domínio foram todos acima de 0,70. 0 valor por subescala foram todos acima de 0,7 exceto nas subescalas de "Interação social com irmãos e irmãs" e "Interação social com jovens". A Tabela 2 apresenta medidas de alfa de Cronbach para cada domínio e para o questionário completo (69 questões do tipo Likert).

A Tabela 3 mostra o valor de alfa de Cronbach em cada subescala na versão final em português e na versão original. ${ }^{(17)}$
Tabela 2. Valores do alfa de Cronbach por domínio

\begin{tabular}{cc}
\hline Domínio & $\alpha$ de Cronbach \\
\hline A. Funcionamento psicológico & 0,810 \\
B. Estado funcional & 0,955 \\
C. Funcionamento físico & 0,839 \\
D. Funcionamento social & 0,796 \\
Formulário todo (69 questões) & 0,906 \\
\hline
\end{tabular}

Tabela 3. Valores do alfa de Cronbach por subescala

\begin{tabular}{|c|c|c|c|}
\hline Domínios & Subescala & $\begin{array}{c}\alpha \text { da versão } \\
\text { final em } \\
\text { português }\end{array}$ & $\begin{array}{c}\alpha \text { da versão } \\
\text { original } \\
\text { (Langeveld, } \\
\text { 1996) }\end{array}$ \\
\hline \multirow{7}{*}{$\begin{array}{l}\text { A. Funcionamento } \\
\text { Fisiológico }\end{array}$} & Stress & 0,781 & 0,85 \\
\hline & Harmonia & 0,758 & 0,82 \\
\hline & Fadiga & 0,719 & 0,80 \\
\hline & Força/vitalidade & 0,808 & 0,76 \\
\hline & Depressão & 0,727 & 0,74 \\
\hline & Bom humor & 0,713 & 0,73 \\
\hline & $\begin{array}{l}\text { Otimismo com } \\
\text { o futuro }\end{array}$ & 0,718 & 0,82 \\
\hline \multirow[t]{2}{*}{$\begin{array}{l}\text { B. Estado } \\
\text { Funcional }\end{array}$} & $\begin{array}{l}\text { Impacto da cefaleia } \\
\text { nas atividades } \\
\text { diárias }\end{array}$ & 0,931 & 0,87 \\
\hline & $\begin{array}{l}\text { Impacto da cefaleia } \\
\text { nas atividades de } \\
\text { lazer }\end{array}$ & 0,890 & 0,82 \\
\hline $\begin{array}{l}\text { C. Funcionamento } \\
\text { Físico }\end{array}$ & $\begin{array}{l}\text { Sintomas } \\
\text { somáticos }\end{array}$ & 0,766 & 0,84 \\
\hline \multirow[t]{3}{*}{$\begin{array}{l}\text { D. Funcionamento } \\
\text { Social }\end{array}$} & $\begin{array}{l}\text { Funcionamento em } \\
\text { casa e na escola }\end{array}$ & 0,785 & 0,68 \\
\hline & $\begin{array}{l}\text { Interação social } \\
\text { com irmãos e irmãs }\end{array}$ & $0,454^{(1)}$ & 0,66 \\
\hline & $\begin{array}{l}\text { Interação social } \\
\text { com jovens }\end{array}$ & 0,651 & 0,72 \\
\hline
\end{tabular}

(1): $O \propto$ de Cronbach é igual a 0,779 se o item 26 for excluído da subescala

\section{DISCUSSÃO}

No presente estudo, a tradução e a retrotradução do QLH-Y para a língua portuguesa foram realizadas de maneira cuidadosa e satisfatória, com o propósito de adaptar esse instrumento para a língua portuguesa, respeitando os valores socioculturais da população brasileira de adolescentes. Foi realizado o mínimo de alteração na estrutura do instrumento original, mantendoos itens da escala, a fim de não promover maiores alterações das propriedades psicométricas e permitindo a comparação das duas versões.

Nos itens: "Vigoroso", "Eu me senti depressivo" e "Rigidez muscular", houve uma mudança na tentativa de facilitar o entendimento dos termos. A incompreensão dos itens pode estar associada a limitações intelectuais. Houve adequação dos termos para "Ativo", "Eu me senti 
triste" e "Tensão muscular". Após as mudanças, o número de indivíduos com dúvidas reduziu consideravelmente.

No item "Se houve atividades em casa, em que todos nós pudéssemos fazê-las juntas, eu também participava" um número significativo de indivíduos entrevistados não compreendeu a questão. Optou-se por transformá-lo em "Participei de atividades na minha casa em que todos puderam participar", tornando a questão mais clara.

Como exemplo da intensidade de correlação entre os itens de um questionário, pode-se verificar se esse coeficiente aumenta depois de eliminar um item da escala de medição (questionário). Se isso ocorrer, pode-se assumir que esse item não é altamente correlacionado com os outros itens da escala. Por outro lado, se o coeficiente diminuir pode ser assumido que esse item é altamente correlacionado com os outros itens da escala. Dessa forma, o alfa de Cronbach determina se a escala é realmente confiável, pois avalia como cada item reflete sua confiabilidade. Após a eliminação do item 26 (Eu tive uma discussão com um dos meus irmãos ou irmãs), na subescala "Interação social com irmãos e irmãs", houve uma elevação do valor do alfa de Cronbach de 0,454 para 0,779 , mostrando que esse item não é altamente correlacionado com os outros itens da escala, justificando a sua exclusão.

Muitos pesquisadores avaliam a validade de um instrumento pelo seu nível de confiabilidade. Conceitualmente, a confiabilidade reflete o quanto os valores observados estão correlacionados aos verdadeiros valores. ${ }^{(20)}$ Um instrumento é confiável se ele mede consistentemente as condições que poderiam causar erros. Existem três tipos de Confiabilidade: interexaminadores (inter-rater), teste-reteste (intraexaminadores) e consistência interna. Este estudo realizou a avaliação da consistência interna.

Os resultados encontrados na consistência interna do presente estudo foram satisfatórios, com o valor de alfa de Cronbach 0,906 (superior a 0,7). Os achados revelam um valor elevado e, portanto, um grau elevado de consistência interna.

Comparando-se com os valores encontrados da consistência interna da versão original em inglês, observase uma redução do valor de alfa de Cronbach de 0,72 para 0,651 na subescala "Interação social com jovem".

O QLH-Y também foi traduzido e validado na ltália. ${ }^{(19)}$ A análise dos dados da versão italiana foi realizada de modo a obter uma redução de itens mantendo a consistência interna máxima. Esta versão possui 52 itens divididos em 9 subescalas que avaliam 4 domínios da
Qualidade de vida. $\bigcirc$ valor do alfa de Cronbach foi superior a 0,70 em todas as subescalas, exceto nas de Depressão $(0,66)$, Sintomas somáticos $(0,64)$, Interação social com irmão e irmãs $(0,65)$ e Funcionamento em casa e na escola $(0,53)$. Estas duas últimas estão incluídas no domínio Funcionamento Social.

As subescalas com valores alfa de Cronbach abaixo de 0,70 foram: Interação social com irmãos $(0,454)$ e irmãs e Interação social com jovens $(0,651)$. Ambas pertencem ao domínio Funcionamento social.

O item 26, "Eu tive uma discussão com um dos meus irmãos ou irmãs," pode não ter se correlacionado com os outros itens devido a uma incompreensão da questão ou devido ao fato de muitos adolescentes não terem irmãos ou não possuírem contato com estes.

As medidas de qualidade de vida podem amparar os estudos de custo benefício identificando quais intervenções devem ser priorizadas. Existe a necessidade de criação e utilização de instrumentos de avaliação da qualidade de vida que valorizem as experiências das crianças e dos adolescentes de maneira adequada a sua fase de desenvolvimento.

O questionário QLH-Y foi devidamente traduzido e adaptado transculturalmente para a língua portuguesa. O questionário QLH-Y apresentou uma consistência interna bastante satisfatória, tanto para as 69 questões quanto para os domínios separadamente. A consistência interna do QLH-Y, medida pelo coeficiente alfa de Cronbach padronizado, foi satisfatória. Obteve-se um índice geral de 0,906, cujo valor excede o valor mínimo de referência.

\section{REFERÊNCIAS}

1. Stovner LJ, Zwart JA, Hagen K, Terwindt GM, Pascual J. Epidemiology of headache in Europe. Eur J Neurol. 2006; 13(4):333-45.

2. Roth-Isigkeit A, Thyen U, Stoven H, Schwarzenberger J, Shumucker P. Pain among children and adolescents: restrictions in daily living and triggering factors. Pediatrics. 2005;1 15(2): 152-62.

3. Bandell-Hoekstra I, Abu-Saad HH, Passchier J, Knipschild P. Recurrent headache, coping, and quality of life in children: a review. Headache. 2000;40(5):357-70.

4. World Health Organization. Constitution of the World Health Organization Basic Documents. Geneva, Switzerland: World Health Organization; 1948.

5. Varni JW, Seid M, Rode CA. The PedsQL: measurement model for the pediatric quality of life inventory. Med Care. 1999;37(2):126-39.

6. Albuquerque RP, Santos AB, Tognola WA,Arruda MA. An epidemiologic study of headaches in Brazilian schoolchildren with a focus on pain frequency. Arq. Neuropsiquiatr. 2009; 67(3-B):798-803. 
7. Slater S, Crawford MJ, Kabbouche MA, LeCates SL, Cherney S, Vaughan P. Effects of gender and age on paediatric headache. Cephalalgia. 2009;29(9):969-73.

8. Gorayeb MA, Gorayeb R. Association between headache and anxiety disorders indicators in a school sample from Ribeirão Preto, Brazil. Arq Neuropsiquiatr. 2002 Sep;60(3-B):764-8. [Article in Portuguese]

9. Barea LM, Tannhauser M, Rotta NT. An epidemiologic study of headache among children and adolescents of southern Brazil. Cephalalgia. 1996;16(8):545-9.

10. Brattberg J. The incidence of back pain and headache among Swedish School children. Qual Life Res. 1994 Dec;3 Suppl 1:S27-31

11. Fendrich K, Vennemann M, Pfaffenrath V, Evers S, May A, Berger K, Hoffmann W. Headache prevalence among adolescents - the German DMKG headache study. Cephalalgia. 2007 Apr; 27(4):347-54.

12. Passchier J. Qualidade de vida na criança com cefaleia. In M.A. Arruda \& V. Guidetti (Eds.), Cefaleias na Infância e Adolescência (pp. 69-73). São Paulo, Brazil, Instituto Glia: 2007.

13. Pakalnis A, Butz C, Splaingard D, Kring D, Fong J. Emotional problems and prevalence of medication overuse in pediatric chronic daily headache. J Child Neurol. 2007 Dec;22(12):1356-9.
14. Puca F, Genco S, Prudenzano MP, Savarese M, Bussone G, D'Amico $D$, et al. Psychiatric comorbidity and psychosocial stress in patientswith tension-type headache from headache centers in Italy. The Italian Collaborative Group for the Study of Psychopathological Factors in Primary Headaches. Cephalalgia. 1999; 19(3):159-64.

15. Zencir M, Ergin H, Sahiner T, Kiliç I, Alkis E, Ozdel L, Gürses D, Ergin A. Epidemiology and symptomatology of migraine among school children: Denizli urban area in Turkey. Headache . 2004; 44(8):780-5.

16. Bandell-Hoekstra I, Abu-Saad HH, Passchier J, Knipschild P, Recurrent headache, coping, and quality of life in children: a review. Headache. 2000;40(5):357-70.

17. Langeveld JH, Koot HM, Loonen MC, Hazebroek-Kampschreur AA, Passchier J.A Quality of Life Instrument for Adolescents with Chronic Headache. Cephalalgia. 1996; 16(3):183-96.

18. WHO. World Health Organization. Pregnant adolescents: delivering on global promises of hope. 2006:1-28.

19. Beaton DE, Bombadier C, Guillemin F, Ferraz MB.Guidelines for the process of cross-cultural adaptation of self-reported measures. Spine. 2000 Dec 15;25(24):3186-91.

20. Streiner DL. A checklist for evaluating the usefulness of rating scales. Can J Psychiatry. 1993 Mar;38(2): 140-8.

21. Nodari E, Battistella PA, Naccarella C, Vidi M. Quality of life in young Italian patients with primary. Headache. 2002; 42(4):268-74.

\section{Anexo 1 \\ Questionário Quality of Life Headache-Youth - QLH-Y Versão em português do Brasil}

Nome: Idade:

Data do preenchimento deste questionário:

Responda cada uma das questões com sua opinião própria. Você pode não ter mais que uma resposta por questão. É muito importante que você responda TODAS as questões.

Quanta satisfação que você teve semana passada em relação a sua vida em geral?

Dê sua resposta para esta questão desenhando um "X" na linha. O quanto mais satisfeito você esteve em relação a sua vida, mais para a direita você deve desenhar o "X". Quanto mais insatisfeito você esteve em relação a sua vida, mais para a esquerda você deve desenhar O "X".

EXEMPLO:

Quanta satisfação que você teve semana passada em relação aos programas de televisão?

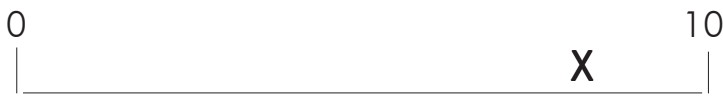
Completamente
Completamente
Insatisfeito
satisfeito

AGORA RESPONDA:

Quanta satisfação você teve semana passada com sua vida em geral?

Marque um "X" na linha abaixo.

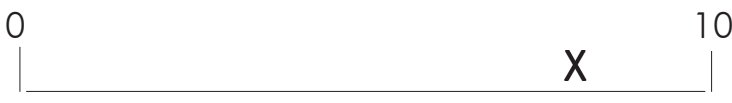

Completamente

insatisfeito
Completamente

satisfeito 
Nós gostaríamos de saber a frequência de cada uma destas situações aplicadas a você na SEMANA PASSADA. Circule ao redor da resposta que se encaixa mais próximo da sua situação.

EXEMPLO:

Se você achou que na semana passada aquele programa de televisão foi "várias vezes ou sempre" interessante, desenhe um círculo ao redor do número 3.

Semana passada, eu achei os programas de televisão:

$\begin{array}{lll}\text { "raramente } & \text { "as vezes" } & \text { "muitas } \\ \text { ou nunca } & \text { vezes" } & \text { vezes ou } \\ & & \text { sempre" }\end{array}$

Interessante 013

\section{RESPONDA}

Semana passada eu estava me sentindo:

\begin{tabular}{|l|l|c|c|c|c|}
\hline 1. & Esgotado & $\begin{array}{c}\text { Raramente } \\
\text { ou nunca }\end{array}$ & Ás vezes & $\begin{array}{c}\text { Muitas } \\
\text { vezes }\end{array}$ & $\begin{array}{c}\text { Várias vezes ou } \\
\text { sempre }\end{array}$ \\
\hline 2. & Nervoso & 0 & 1 & 2 & 3 \\
\hline 3. & Apavorado & 0 & 1 & 2 & 3 \\
\hline 4. & Cansado & 0 & 1 & 2 & 3 \\
\hline 5. & Tenso & 0 & 1 & 2 & 3 \\
\hline 6. & No final da minha força & 0 & 1 & 2 & 3 \\
\hline 7. & Tranquilo & 0 & 1 & 2 & 3 \\
\hline 8. & Preocupado & 0 & 1 & 2 & 3 \\
\hline 9. & Insignificante & 0 & 1 & 2 & 3 \\
\hline 10. & Bem & 0 & 1 & 2 & 3 \\
\hline 11. & Desgastado & 0 & 1 & 2 & 3 \\
\hline 12. & Ansioso & 0 & 1 & 2 & 3 \\
\hline 13. & Satisfeito & 0 & 1 & 2 & 3 \\
\hline 14. & Feliz & 0 & 1 & 2 & 3 \\
\hline 15. & Incerto & 0 & 1 & 2 & 3 \\
\hline 16. & Desesperado & 0 & 1 & 2 & 3 \\
\hline 17. & Cheio de energia & 0 & 1 & 2 & 3 \\
\hline 18. & Ativo & 0 & 1 & 2 & 3 \\
\hline 19. & Forte & 0 & 1 & 2 & 3 \\
\hline 20. & Relaxado & 1 & 2 & 3 \\
\hline
\end{tabular}

Dê uma resposta que se encaixe mais próximo da sua situação SEMANA PASSADA:

\begin{tabular}{|c|c|c|c|c|c|}
\hline & & $\begin{array}{c}\text { Raramente } \\
\text { ou nunca }\end{array}$ & Às vezes & $\begin{array}{l}\text { Muitas } \\
\text { vezes }\end{array}$ & $\begin{array}{c}\text { Várias vezes ou } \\
\text { sempre }\end{array}$ \\
\hline 21. & Participei de atividade na minha casa em que todos puderam participar. & 0 & 1 & 2 & 3 \\
\hline 22. & Eu me dei bem com (um dos) meus pais & 0 & 1 & 2 & 3 \\
\hline 23. & Eu poderia falar com (um dos) meus pais sobre coisas pessoais ou problemas & 0 & 1 & 2 & 3 \\
\hline 24. & Eu me dei bem com (um dos) meus irmãos ou irmãs & 0 & 1 & 2 & 3 \\
\hline 25. & Eu poderia falar com (um dos) meus irmãos & 0 & 1 & 2 & 3 \\
\hline 26. & Eu me diverti com jovens da minha idade & 0 & 1 & 2 & 3 \\
\hline
\end{tabular}


(Continuação)

\begin{tabular}{|c|c|c|c|c|c|}
\hline & & $\begin{array}{c}\text { Raramente } \\
\text { ou nunca }\end{array}$ & Às vezes & $\begin{array}{l}\text { Muitas } \\
\text { vezes }\end{array}$ & $\begin{array}{l}\text { Várias vezes ou } \\
\text { sempre }\end{array}$ \\
\hline 27. & Eu poderia falar com um amigo sobre coisas pessoais ou problemas & 0 & 1 & 2 & 3 \\
\hline 28. & $\begin{array}{l}\text { Eu fiz algumas atividades com jovens da minha idade, como esportes, ir ao } \\
\text { cinema, sair etc. }\end{array}$ & 0 & 1 & 2 & 3 \\
\hline 29. & Meus resultados escolares ou resultados no trabalho foram bons & 0 & 1 & 2 & 3 \\
\hline 30. & Eu resolvi meu dever (de casa) a tempo & 0 & 1 & 2 & 3 \\
\hline 31. & Eu me diverti na escola ou no trabalho & 0 & 1 & 2 & 3 \\
\hline 32. & Eu não dormi bem & 0 & 1 & 2 & 3 \\
\hline 33. & Eu não senti vontade de fazer nada & 0 & 1 & 2 & 3 \\
\hline 34. & Eu me senti depressivo & 0 & 1 & 2 & 3 \\
\hline 35. & Quando eu acordei, eu pensei sobre coisas boas a vir & 0 & 1 & 2 & 3 \\
\hline 36. & Eu não me senti bem & 0 & 1 & 2 & 3 \\
\hline 37. & Eu pensei que tenho uma boa vida & 0 & 1 & 2 & 3 \\
\hline 38. & $\begin{array}{l}\text { Eu pensei que no futuro eu teria uma boa casa e um bom emprego e seria } \\
\text { capaz de conseguir dinheiro suficiente }\end{array}$ & 0 & 1 & 2 & 3 \\
\hline 39. & Eu pensei que tudo vai ficar bem & 0 & 1 & 2 & 3 \\
\hline 40. & Eu me senti alegre & 0 & 1 & 2 & 3 \\
\hline 41. & $\begin{array}{l}\text { Eu pensei que eu jamais serei alguém no futuro porque eu não sou bom em } \\
\text { nada }\end{array}$ & 0 & 1 & 2 & 3 \\
\hline 42. & Eu pensei que eu iria ser feliz no futuro & 0 & 1 & 2 & 3 \\
\hline 43. & $\begin{array}{l}\text { Eu tive a sensação de eu não me adapto quando os jovens da minha idade } \\
\text { estão ocupados com de algo bom }\end{array}$ & 0 & 1 & 2 & 3 \\
\hline 44. & Geralmente, eu estava de bom humor & 0 & 1 & 2 & 3 \\
\hline
\end{tabular}

Responda esta questão do mesmo jeito que a primeira no começo deste questionário:

Marque um "X" na linha.

\section{RESPONDA:}

O quanto você esteve satisfeito com sua saúde na semana passada?

Completamente

insatisfeito

Completamente

satisfeito

SEMANA PASSADA eu estava incomodado por:

\begin{tabular}{|l|l|c|c|c|c|}
\hline & & $\begin{array}{c}\text { Raramente } \\
\text { ou nunca }\end{array}$ & Às vezes & $\begin{array}{c}\text { Muitas } \\
\text { vezes }\end{array}$ & $\begin{array}{c}\text { Várias vezes ou } \\
\text { sempre }\end{array}$ \\
\hline 45. & Dor de barriga & 0 & 1 & 2 & 3 \\
\hline 46. & Dificuldade para dormir & 0 & 1 & 2 & 3 \\
\hline 47. & Me sentir cansado & 0 & 1 & 2 & 3 \\
\hline 48. & $\begin{array}{l}\text { Sentir que eu ia ficar doente } \\
\text { (enjoado) }\end{array}$ & 0 & 1 & 2 & 3 \\
\hline 49. & Tensão muscular & 0 & 1 & 2 & 3 \\
\hline 50. & $\begin{array}{l}\text { Sentir que meu coração } \\
\text { estava batendo muito rápido }\end{array}$ & 0 & 1 & 2 & 3 \\
\hline 51. & Dor nos olhos ao ler & 0 & 1 & 2 & 3 \\
\hline 52. & Sentir tontura & 0 & 1 & 2 & 3 \\
\hline 53. & Dores de cabeça & 0 & 1 & 2 & 3 \\
\hline 54. & Me sentir fraco & 0 & 1 & 2 & 3 \\
\hline
\end{tabular}


As questões seguintes são relacionadas com o modo que as dores de cabeça interferiram em você na semana passada. Se você NÃO SENTIU DOR DE CABEÇA na semana passada. Neste caso, desenhe círculos ao redor do número 0.

Para responder as questões, desenhe um círculo ao redor de um dos quatros números no final de cada questão. Os números agora significam:

$0=$ "de jeito nenhum"; 1 ="pouco"; 2 = "mais ou menos"; $3=$ "muito".

\begin{tabular}{|l|l|c|c|c|c|}
\hline & & $\begin{array}{c}\text { De jeito } \\
\text { nenhum }\end{array}$ & Pouco & $\begin{array}{c}\text { Mais ou } \\
\text { menos }\end{array}$ & Muito \\
\hline 55. & Meu passatempo & 0 & 1 & 2 & 3 \\
\hline 56. & Meu trabalho & 0 & 1 & 2 & 3 \\
\hline 57. & Meus Passeios & 0 & 1 & 2 & 3 \\
\hline 58. & Minha relação com meus pais, ou irmãos \\
e irmãs & 0 & 1 & 2 & 3 \\
\hline 59. & Minha relação com meus amigos & 0 & 1 & 2 & 3 \\
\hline 60. & Meu sono ou meus horários para dormir & 0 & 1 & 2 & 3 \\
\hline 61. & Meu apetite & 0 & 1 & 2 & 3 \\
\hline 62. & Minha habilidade em ler & 0 & 1 & 2 & 3 \\
\hline 63. & Memorizar alguma coisa que eu tenha lido & 0 & 1 & 2 & 3 \\
\hline 64. & Pensar sobre as coisas & 0 & 1 & 2 & 3 \\
\hline 65. & Escutar música & 0 & 1 & 2 & 3 \\
\hline 66. & Andar de bicicleta & 0 & 1 & 2 & 3 \\
\hline 67. & Fazer atividades esportivas & 0 & 1 & 2 & 3 \\
\hline 68. & Assistir televisão & 0 & 1 & 2 & 3 \\
\hline
\end{tabular}

Este é o fim do questionário. Por favor, confira cuidadosamente se você não esqueceu nenhuma questão ou deu mais de uma resposta a uma questão. Obrigado pela sua colaboração.

Conflito de interesse: nada a declarar. Fonte financiadora do projeto: Recursos próprios

Recebido em 5 de março 2013

Aceito em 27 de maio 2013

Correspondência

Daniella Araújo de Oliveira Av. Jorn. Anibal Fernandes, s/n, Cidade Universitária, 50740-560 - Recife, PE, Brasil Fone:(55-81) 21268937, Fax: (55-81) 21268491 sabino_daniella@ig.com.br 